\title{
A Note on the Adversary Degree Associated Reconstruction Number of Graphs
}

\author{
S. Monikandan, ${ }^{1}$ S. Sundar Raj, ${ }^{2}$ C. Jayasekaran, ${ }^{3}$ and A. P. Santhakumaran ${ }^{4}$ \\ ${ }^{1}$ Department of Mathematics, Manonmaniam Sundaranar University, Tirunelveli 627 012, India \\ ${ }^{2}$ Department of Mathematics, Vivekananda College, Agasteeswaram 629 701, India \\ ${ }^{3}$ Department of Mathematics, Pioneer Kumaraswamy College, Nagercoil 629 003, India \\ ${ }^{4}$ Department of Mathematics, Hindustan Institute of Technology and Science, Hindustan University, Chennai 103, India
}

Correspondence should be addressed to S. Monikandan; monikandans@gmail.com

Received 8 November 2012; Accepted 14 March 2013

Academic Editor: Leonidas Palios

Copyright (C) 2013 S. Monikandan et al. This is an open access article distributed under the Creative Commons Attribution License, which permits unrestricted use, distribution, and reproduction in any medium, provided the original work is properly cited.

A vertex-deleted subgraph of a graph $G$ is called a card of $G$. A card of $G$ with which the degree of the deleted vertex is also given is called a degree associated card (or dacard) of $G$. The degree associated reconstruction number drn $(G)$ of a graph $G$ is the size of the smallest collection of dacards of $G$ that uniquely determines $G$. The adversary degree associated reconstruction number of a graph $G$, $\operatorname{adrn}(G)$, is the minimum number $k$ such that every collection of $k$ dacards of $G$ that uniquely determines $G$. In this paper, we show that adrn of wheels and complete bipartite graphs on at least 4 vertices is 2 or 3 .

\section{Introduction}

All graphs considered are simple, finite, and undirected. We will mostly follow the standard graph theoretic terminology of [1]. A vertex-deleted subgraph or card $G-v$ of a graph $G$ is the unlabeled graph obtained from $G$ by deleting the vertex $v$ and all edges incident with $v$. The ordered pair $(d(v), G-$ $v$ ) is called a degree associated card or dacard of the graph $G$ where $d(v)$ is the degree of $v$ in $G$. The deck (dadeck) of a graph $G$ is its collection of cards (dacards). Ulam's Conjecture [2], also called Reconstruction Conjecture (RC), asserts that every graph on at least three vertices is determined uniquely (up to isomorphism) by its deck. Graphs that obey RC are called reconstructible.

For a reconstructible graph $G$, Harary and Plantholt [3] have defined the reconstruction number $r n(G)$ to be the size of the smallest subcollection of the deck of $G$ which is not contained in the deck of any other graph $H ; H \not$ G. Myrvold [4] referred to this number as ally-reconstruction number of G. Myrvold [5] also studied adversary reconstruction number of $G$ which is the smallest $k$ such that no subcollection of the deck of $G$ of size $k$ is contained in the deck of any other graph $H ; H \neq G$.
An extension of RC to digraphs, the Digraph Reconstruction Conjecture, was disproved when Stockmeyer exhibited [6] several infinite families of counter-examples. In view of this, Ramachandran [7] studied the degree (degree triple) associated reconstruction of graphs (digraphs). For a vertex $v$ of a digraph, the ordered triple $(r, s, t)$ is called the degree triple of $v$ where $r, s$, and $t$ are, respectively, the number of unpaired outarcs, unpaired inarcs, and symmetric pairs of arcs incident with $v$. A graph (digraph) is called degree associated reconstructible if it can be determined uniquely from its dadeck. For a degree associated reconstructible graph (digraph) $G$, the degree (degree triple) associated reconstruction number, $\operatorname{drn}(G)$, of $G$ is the size of the smallest subcollection of the dadeck of $G$ which is not contained in the dadeck of any other graph (digraph) $H ; H \neq$ G. Barrus and West [8] have shown that $\operatorname{drn}(G)=2$ for all caterpillars except stars and one 6-vertex example, and that $\operatorname{drn}(G) \geq 3$ for all vertex-transitive graphs $G$ (not complete or edgeless).

The following weakening of the reconstruction problem has also been considered by Harary and Plantholt [3]. A graph $G$, in a given class of graphs $\mathscr{C}$, is called class-reconstructible if whenever $H \in \mathscr{C}$ has the same deck as $G$, then $G \cong$ $H$. If a graph is degree associated reconstructible then it is 
class-reconstructible, and vice-versa, where the class is the class of graphs with a given number $m$ of edges.

In this paper, we study the parameter adversary degree associated reconstruction number $\operatorname{adrn}(G)$ of a graph $G$. For a reconstructible graph $G$ from its dadeck, $\operatorname{adrn}(G)$ is the minimum number $k$ such that every collection of $k$ dacards of $G$ is not contained in the dadeck of any other graph $H ; H \not \equiv$ $G$. From their definitions, it is clear that $\operatorname{drn}(G) \leq \operatorname{adrn}(G)$ for any graph $G$; the equality holds for vertex-transitive graphs (where all the dacards are necessarily identical). In this paper, we show that $a d r n$ is 2 or 3 for wheels and complete bipartite graphs on at least 4 vertices.

\section{2. adrn of Standard Graphs}

Since $\operatorname{drn}(G)$ and $\operatorname{adrn}(G)$ are exactly equal for any vertextransitive graph $G$, the next theorem follows from [7].

Theorem 1. (i) $\operatorname{adrn}\left(K_{n}\right)=1$.

(ii) $\operatorname{adrn}\left(K_{n, n}\right)=3$ for $n>1$.

(iii) $\operatorname{adrn}\left(C_{n}\right)=3$ for $n \geq 4$, where $C_{n}$ is a cycle on $n$ vertices.

It is clear, from the definition of $\operatorname{adrn}$, that $\operatorname{adrn}\left(K_{n}\right)=$ $\operatorname{adrn}\left(\bar{K}_{n}\right)=1$. In fact, it is true that the $\operatorname{adrn}$ of the complement of a graph is equal to the $a d r n$ of the graph.

Lemma 2. For any $\operatorname{graph} G, \operatorname{adrn}(\bar{G})=\operatorname{adrn}(G) \geq \operatorname{drn}(G)$.

Proof. The latter inequality follows immediately from the definitions of $d r n$ and $a d r n$. To prove the first equality, let $G$ be a graph of order $n$; let $\operatorname{adrn}(G)=s$. Then, there exists a graph $H(\not G)$ such that $G$ and $H$ have $s-1$ dacards in common. If $\left(d_{i}, G_{i}\right)$ is a dacard of $G$, then $\left(n-1-d_{i}, \bar{G}_{i}\right)$ is a dacard of $\bar{G}$ and vice-versa. The graph $\bar{H}(\not \bar{G})$ has therefore $s-1$ dacards in common with those of $\bar{G}$. Consequently, we have $\operatorname{adrn}(\bar{G}) \geq s$.

On the other hand, if $\operatorname{adrn}(\bar{G})>s$, then there exists a graph $T(\nexists \bar{G})$ such that $T$ has $s$ dacards of $\bar{G}$. It follows that the graph $\bar{T}(\not G)$ has $s$ dacards of $G$. Therefore, $\operatorname{adrn}(G)>s$, giving a contradiction. Hence, $\operatorname{adrn}(\bar{G})=s=\operatorname{adrn}(G)$.

An extension of a dacard $(d(v), G-v)$ of $G$ is a graph obtained from the dacard by adding a new vertex $x$ and joining it with $d(v)$ vertices of the dacard, and it is denoted by $H(d(v), G-v)$ (or simply by $H$ ). Throughout this paper, $H$ and $x$ are used in the sense of this definition. follows.

For a graph $G$, to prove $\operatorname{adrn}(G)=k$, we proceed as

(i) First, find the dadeck of $G$.

(ii) Determine next all possible extensions of every dacard of $G$.

(iii) Finally, show that every extension other than $G$ has at most $k-1$ dacards in common with those of $G$, and that at least one extension has precisely $k-1$ dacards in common with those of $G$.
A vertex of degree $m$ is called an $m$-vertex. We call a neighbour of degree $r$ of a vertex $v$ by an $r$-neighbour of $v$. The union $G \cup H$ of graphs $G$ and $H$ is the graph with vertex set $V(G) \cup V(H)$ and edge set $E(G) \cup E(H)$. The join $G+H$ of disjoint graphs $G$ and $H$ is the graph obtained from $G \cup H$ by joining each vertex of $G$ to each vertex of $H$.

Remark 3. Note that if a dacard of a graph $G$ is vertextransitive and the degree associated with the dacard is one or else the degree associated with the dacard equals the number of vertices in the dacard, then the dacard has a unique extension (up to isomorphism), and, hence, $\operatorname{drn}(G)=1$.

Since the wheel $W_{n}$ on $n(\geq 4)$ vertices has an $(n-1)$ vertex, the dacard (obtained by deleting the $(n-1)$-vertex of $\left.W_{n}\right)$ has a unique extension, and so $\operatorname{drn}\left(W_{n}\right)=1$ by Remark 3 . We now show that $\operatorname{adr} n\left(W_{n}\right)=3$ for $n>4$.

Theorem 4. If $W_{n}$ is a wheel on $n(\geq 4)$ vertices, then

$$
\operatorname{adrn}\left(W_{n}\right)= \begin{cases}1, & \text { if } n=4, \\ 3, & \text { otherwise. }\end{cases}
$$

Proof. The dadeck of $W_{n}=K_{1}+C_{n-1}$ consists of one copy of the dacard $\left(n-1, C_{n-1}\right)$ and $n-1$ copies of the dacard $\left(3, K_{1}+\right.$ $\left.P_{n-2}\right)$, where $v$ is a vertex of $C_{n-1}$. Let $H\left(3, K_{1}+P_{n-2}\right)$ be an extension of the dacard $\left(3, K_{1}+P_{n-2}\right)$. We consider four cases depending on the value of $n$.

Case 1. $n=4$. Now, all the four dacards are isomorphic and they are $\left(3, C_{3}\right)$. Since it has a unique extension, it follows that $\operatorname{adrn}\left(W_{4}\right)=1$.

Case 2. $n=5$. In the dacard $\left(3, K_{1}+P_{3}\right)$, there are two 2vertices and two 3 -vertices. If we join the newly added vertex $x$ to the two 2 -vertices and a 3-vertex, then the extension $H$ is isomorphic to $W_{n}$. Therefore, we join the newly added vertex $x$ to a 2 -vertex and the two 3 -vertices. The extension $H$ so has one 2-vertex, two 3 -vertices, and two 4 -vertices (Figure 1). The dacard of the extension $H$ corresponding to each of the two 3 -vertices is $\left(3, K_{1}+P_{3}\right)$. The extension $H$ has thus only two dacards in common to those of $W_{n}$, and, hence, $\operatorname{adrn}\left(W_{5}\right)=3$.

Case 3. $n=6$. In the dacard $\left(3, K_{1}+P_{4}\right)$, there are exactly two 2 -vertices, one 4 -vertex, and two 3 -vertices.

Case 3.1. Join $x$ to none of the two 2 -vertices.

The extension $H$ has one vertex of degree 3, two vertices of degree 2, two vertices of degree 4 , and one vertex of degree 5 (Figure 2). The dacard corresponding to the 3-vertex of the extension $H$ is clearly in common with that of $W_{n}$. The dacard of $H$ obtained by deleting the 5-vertex has endvertices, and, hence, it is not a dacard of $W_{n}$. The extension $H$ has thus only one dacard in common with that of $W_{n}$.

Case 3.2. Join $x$ to one 2-vertex and two 3 -vertices. 


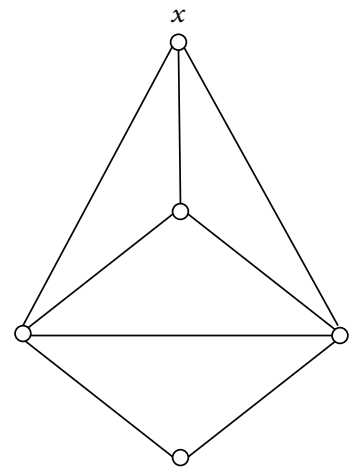

FIgure 1: The extension $H$ in Case 2.

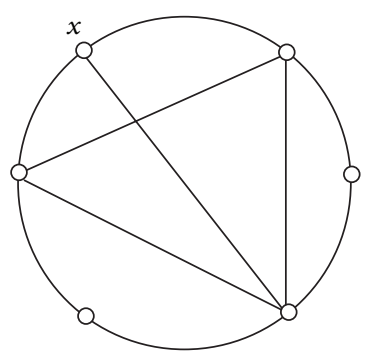

Figure 2: The extension $H$ in Case 3.1.

The extension $H$ has two vertices of degree 3, one vertex of degree 2, and three vertices of degree 4 . The dacard corresponding to each of the two 3-vertices is a dacard of $W_{n}$. The extension $H$ has thus only two dacards in common with those of $W_{n}$.

Case 3.3. Join $x$ to one 2 -vertex (say $y$ ), one 3-vertex (say $z$ ), and the unique 4 -vertex.

Case 3.3.1. Vertices $y$ and $z$ are nonadjacent.

In $H, y$ is a 3-vertex and $z$ is a 4-vertex. The dacards of $H$ corresponding to $x$ and the 3-vertex other than $y$ are dacards of $W_{n}$. The dacard corresponding to $y$ of $H$ is not a dacard of $W_{n}$, since the subgraph of $H$ obtained by deleting $y$ has three 2 -vertices. The dacard corresponding to the 5-vertex of $H$ is not a dacard of $W_{n}$. The extension $H$ has thus only two dacards in common with those of $W_{n}$.

Case 3.3.2. Vertices $y$ and $z$ are adjacent.

The dacards corresponding to the 3-vertices $x$ and $y$ in $H$ are dacards of $W_{n}$. The dacard corresponding to the other 3 -vertex is not a dacard of $W_{n}$, since the 3-vertex is adjacent to a 2 -vertex in $H$. Also in $H$, the 5 -vertex is adjacent to a 2 vertex, and so the dacard corresponding to the 5-vertex is not a dacard of $W_{n}$. The extension $H$ has thus only two dacards in common with those of $W_{n}$.

Case 3.4. Join $x$ to two 2 -vertices and a 3 -vertex or 4 -vertex (say $y$ ).

If $y$ is the 4-vertex, then $H$ is isomorphic to $W_{n}$. Otherwise, $H$ has four 3 -vertices and two 4 -vertices. The dacards of $H$ corresponding to $x$ and the 3 -vertex $z$ not adjacent to $y$ are dacards of $W_{n}$. But the dacard obtained from $H$ by deleting a 3 -vertex other than $x$ and $z$ has no 4 -vertex, and so it is not a dacard of $W_{n}$. Thus, $H$ has only two dacards in common with those of $W_{n}$.

Case 4. $n \geq 7$. The dacard $\left(3, K_{1}+P_{n-2}\right)$ has two vertices of degree $2, n-4(\geq 3)$ vertices of degree 3 , and one vertex of degree $n-2(\geq 5)$. If the newly added vertex $x$ is not joined to the $(n-2)$-vertex, then the extension $H$ cannot have more than one dacard in common with that of $W_{n}$. If $x$ is joined to the $(n-2)$-vertex and the two 2 -vertices, then $H$ is isomorphic to $W_{n}$. Therefore, it is enough to consider the case in which $x$ is joined to the $(n-2)$-vertex and not joined to at least one 2-vertex.

Case 4.1. Join $x$ to the $(n-2)$-vertex, one 2 -vertex, and one 3-vertex.

The extension $H$ has one 2 -vertex, $n-3$ vertices of degree 3 , one vertex of degree 4 , and one vertex of degree $n-1$. The dacard $\left(3, K_{1}+P_{n-2}\right)$ cannot have a vertex of degree 4 . Therefore, only dacards corresponding to the 3-neighbours of the 4-vertex in $H$ can be isomorphic to the dacard $\left(3, K_{1}+\right.$ $\left.P_{n-2}\right)$.

If the 4 -vertex is adjacent to the 2 -vertex in $H$, then there are two 3-vertices adjacent to the 4-vertex. The extension $H$ therefore can have at most two dacards in common with those of $W_{n}$, and the dacard, corresponding to each of the two 3vertices adjacent to the 4-vertex, is a dacard of $W_{n}$. Otherwise, the 3-neighbour closest to the 2-vertex yields a dacard with a cut vertex, while no dacard of $W_{n}$ has a cut vertex.

Case 4.2. Join $x$ to the $(n-2)$-vertex and two 3-vertices.

The extension $H$ has two vertices of degree $2, n-5(\geq 2)$ vertices of degree 3 , two vertices of degree 4 , and one vertex of degree $n-1$. The dacard $\left(3, K_{1}+P_{n-2}\right)$ cannot have a vertex of degree 4 . Therefore, only dacards corresponding to the 3-vertices which are common neighbours of the two 4 -vertices can be isomorphic to the dacard $\left(3, K_{1}+P_{n-2}\right)$. In the extension $H$, there are at most two 3-vertices which are the common neighbours of the two 4-vertices. Thus, the extension $H$ has at most two dacards in common with those of $W_{n}$, which completes the proof.

From Remark 3, it follows that $\operatorname{drn}\left(K_{1, n}\right)=1$. But $\operatorname{adrn}\left(K_{1, n}\right)$ can be greater than one for $n \geq 3$ by the next theorem.

\section{Theorem 5. For $n \geq 1$}

$$
\operatorname{adrn}\left(K_{1, n}\right)= \begin{cases}1, & \text { if } n \leq 2, \\ 3, & \text { if } n=3, \\ 2, & \text { if } n>3\end{cases}
$$

Proof. The dadeck of $K_{1, n}$ consists of one copy of $\left(n, \bar{K}_{n}\right)$ and $n$ copies of $\left(1, K_{1, n-1}\right)$. The extension $H\left(n, \bar{K}_{n}\right)$ is clearly isomorphic to $K_{1, n}$. Consider the extension $H\left(1, K_{1, n-1}\right)$. If we join the newly added vertex $x$ to the $(n-1)$-vertex of the dacard $\left(1, K_{1, n-1}\right)$, then the extension $H$ is isomorphic 
to $K_{1, n}$. We join therefore the vertex $x$ to any one of the endvertices of the dacard. For $n=2$, the extension $H$ is isomorphic to $K_{1, n}$, and, hence, $\operatorname{adr} n\left(K_{1,2}\right)=1$. For $n=3$, the extension $H$ is isomorphic to $P_{4}$. The dadeck of $P_{4}$ consists of 2 copies of $K_{1,2}$ and 2 copies of $K_{1} \cup K_{2}$. The extension $H$ therefore has exactly two dacards in common with those of $K_{1, n}$ and $\operatorname{adrn}\left(K_{1,3}\right)=3$. Now, let us assume that $n \geq$ 4. Then, in the extension $H$, there is exactly one 2 -vertex which is adjacent to the newly added endvertex and a unique $(n-1)$-vertex. Since there is no 2 -vertex in $K_{1, n-1}$ for $n \geq 4$, only the dacard corresponding to the 1-neighbour of the 2vertex in the extension $H$ can be a dacard of $K_{1, n}$. Hence, $\operatorname{adrn}\left(K_{1, n}\right)=2$.

Ramachandran [7] proved that $\operatorname{drn}\left(K_{m, n}\right)=2$ for $2 \leq$ $m<n$. We shall show that adrn of $K_{m, n}$ is not always two.

Theorem 6. For $2 \leq m<n$,

$$
\operatorname{adrn}\left(K_{m, n}\right)= \begin{cases}3, & \text { if } m=n-2, \\ 2, & \text { otherwise. }\end{cases}
$$

Proof. The dadeck of $K_{m, n}$ consists of $m$ copies of $\left(n, K_{m-1, n}\right.$ ) and $n$ copies of $\left(m, K_{m, n-1}\right)$. To get an extension $H$ having at least one dacard in common with that of $K_{m, n}$, augment the dacard $\left(n, K_{m-1, n}\right)$ or $\left(m, K_{m, n-1}\right)$ by adding a new vertex $x$ and joining it to precisely $n$ or $m$ vertices, respectively, in the dacard.

Case 1. Augmenting the dacard $\left(n, K_{m-1, n}\right)$.

Let $(A, B)$ be the bipartition of the dacard $K_{m-1, n}$, where $|A|=m-1$. Then, if we join $x$ to every vertex of $B$, then $H \cong K_{m, n}$. We join $x$ therefore to at least one vertex of $A$. If we join $x$ to all the $m-1$ vertices of $A$, then no vertex other than $x$ can have degree $n$ in $H$. The extension $H$ therefore has only one dacard isomorphic to $\left(n, K_{m-1, n}\right)$. In this extension, the vertices have degrees $m-1, m, n$, and $n+1$ only. The degrees of the vertices in the dacard corresponding to an $m$-vertex of the extension $H$ are $m-1, m, n-1$ and $n$ where $m-1<m \leq$ $n-1<n$. The dacard $\left(m, K_{m, n-1}\right)$ is therefore not a dacard of the extension $H$. Thus, $H$ has only one dacard in common with that of $K_{m, n}$. If at least one vertex of $A$ is not joined to $x$, then in $H$, there are vertices of degrees $m-1, m, n$, and $n+1$. This extension clearly has a dacard $\left(n, K_{m-1, n}\right)$ (dacard corresponding to the vertex $x$ ). The degrees of the vertices in the dacard corresponding to an $n$-vertex other than $x$ of $H$ are $m-2, m-1, n$, and $n+1$. Therefore, $H$ has only one dacard isomorphic to $\left(n, K_{m-1, n}\right)$. The degrees of the vertices in the dacard corresponding to an $m$-vertex of $H$ are $m-1, m, n-1$, and $n$ where $m-1<m \leq n-1<n$. Hence, this extension $H$ has only one dacard in common with that of $K_{m, n}$.

Case 2. Augmenting the dacard $\left(m, K_{m, n-1}\right)$.

Let $(A, B)$ be the bipartition of the dacard $K_{m, n-1}$, where $A$ is the set of $m$ vertices. If we join $x$ to every vertex of $A$, then the extension $H$ is isomorphic to $K_{m, n}$. We join $x$ therefore to at least one vertex of $B$.
Case 2.1. Join $x$ to no vertex of $A$.

If $n=m+1$, then the extension $H$ is isomorphic to $K_{m, n}$. If $n=m+2$, then the extension $H$ has two dacards isomorphic to $\left(m, K_{m, n-1}\right)$, and, hence, it has only two dacards in common with those of $K_{m, n}$. So, we take that $n>m+2$. Now, the extension $H$ has vertices of degrees $m, m+1$ and $n-1$ only. Clearly, this extension $H$ has a dacard $\left(m, K_{m, n-1}\right)$ corresponding to the $m$-vertex $x$. The removal of any other $m$ vertex from this extension would give a dacard with vertices of degrees $m, m+1$, and $n-2$ only, and, hence, this dacard would not be isomorphic to $K_{m, n-1}$. Thus, $H$ has only one dacard isomorphic to $\left(m, K_{m, n-1}\right)$, and this is the only dacard in common with that of $K_{m, n}$.

Case 2.2. Join $x$ to at least one vertex of $A$.

Case 2.2.1. Join $x$ to exactly one vertex (say $y$ ) of $A$ and exactly one vertex (say $z$ ) of $B$.

In this case, $m=2$ and the extension $H$ contains exactly one triangle, say $x y z$. The dacard of the extension $H$ corresponding to the vertex $x$ is clearly $\left(m, K_{m, n-1}\right)$. The extension $H$ may have two more dacards (corresponding to the vertices $y$ and $z$ ) in common with those of $K_{m, n}$, since no dacard of $K_{m, n}$ contains a triangle. In the extension $H$, there exists at least one m-vertex in $B$ other than $x$. Fix one such vertex and let it be $u$. The $m$-vertex $u$ in the dacard obtained by deleting the vertex $z$ from $H$ is not adjacent to the $(m-1)$ vertex $x$. The dacard of $H$ corresponding to the vertex $z$ is therefore not isomorphic to $\left(n, K_{m-1, n}\right)$ (this verification is needed only for the case when $m+1=n)$. If we remove the vertex $y$ from the extension $H$, then the $(m-1)$-vertex $u$ in the resulting dacard is not adjacent to the $m$-vertex $z$, and, hence, the dacard of $H$ corresponding to the vertex $y$ is not isomorphic to $\left(m, K_{m, n-1}\right)$. The extension $H$ has thus only one dacard in common with that of $K_{m, n}$.

Case 2.2.2. Join $x$ to exactly one vertex (say $y$ ) of $A$ and at least two vertices of $B$.

The extension $H$ has at least two triangles. Clearly, the dacard of $H$ corresponding to the vertex $x$ is $\left(m, K_{m, n-1}\right)$. The extension $H$ may have one more dacard (corresponding to the vertex $y$ ) in common with that of $K_{m, n}$. In $H$, there exists at least one $(n-1)$-vertex in $A$. Fix one such vertex and let it be $z$. Then, the $(n-1)$-vertex $z$ in the dacard of $H$ obtained by deleting the vertex $y$ is not adjacent to the $(m-1)$-vertex $x$, where $m \neq n$. The dacard of $H$ (corresponding to the vertex $y$ ) is therefore not isomorphic to $\left(n, K_{m-1, n}\right)$. The extension $H$ has thus only one dacard in common with that of $K_{m, n}$.

Case 2.2.3. Join $x$ to at least two vertices of $A$ and exactly one vertex (say $y$ ) of $B$.

The extension $H$ has at least two triangles. Clearly, the dacard of $H$ corresponding to the vertex $x$ is $\left(m, K_{m, n-1}\right)$. The extension $H$ may have one more dacard (corresponding to the vertex $y$ ) in common with that of $K_{m, n}$. In $H$, there exists at least one $(n-1)$-vertex of $A$. Fix one such vertex and let it be $z$. The $(n-1)$-vertex $z$ in the dacard of $H$, obtained by deleting the vertex $y$, is then not adjacent to the $(m-1)$-vertex 
TABLE 1: $d r n$ and $a d r n$ of graphs $G$.

\begin{tabular}{lccc}
\hline$G$ & $m, n$ & $\operatorname{drn}(G)$ & $\operatorname{adrn}(G)$ \\
\hline$K_{n}$ & $n>1$ & 1 & 1 \\
$K_{n, n}$ & $n \geq 4$ & 3 & 3 \\
$C_{n}$ & $n=4$ & 3 & 3 \\
$W_{n}$ & $n>4$ & 1 & 1 \\
$W_{n}$ & $n \leq 2$ & 1 & 3 \\
$K_{1, n}$ & $n=3$ & 1 & 1 \\
$K_{1, n}$ & $n>3$ & 1 & 3 \\
$K_{1, n}$ & $2 \leq m<n \neq m+2$ & 1 & 2 \\
$K_{m, n}$ & $2 \leq m, n=m+2$ & 2 & 2 \\
$K_{m, n}$ & 2 & 3 \\
\hline
\end{tabular}

$x$, where $m \neq n$. The dacard of $H$ (corresponding to the vertex $y$ ) is therefore not isomorphic to $\left(n, K_{m-1, n}\right)$. The extension $H$ has thus only one dacard in common with that of $K_{m, n}$.

Case 2.2.4. Join $x$ to at least two vertices of $A$ and at least two vertices of $B$.

Deleting $x$ from $H$ would give a dacard isomorphic to $\left(m, K_{m, n-1}\right)$. The deletion of any vertex other than $x$ from $H$ will give a dacard containing a triangle. The extension $H$ has thus only one dacard isomorphic to $\left(m, K_{m, n-1}\right)$, and this is the only dacard in common with that of $K_{m, n}$, which completes the proof of Theorem 6.

\section{Concluding Remarks}

It is clear, from their definitions, that $\operatorname{drn}(G) \leq \min \{r n(G)$, $\operatorname{adrn}(G)\}$. But $r n(G)$ and $\operatorname{adrn}(G)$ are not comparable in general. For instance, it is proved [9] that $\operatorname{adrn}\left(P_{4}\right)=3$ and $\operatorname{adrn}\left(P_{5}\right)=4$. Therefore, $\operatorname{adrn}\left(P_{4}\right)<r n\left(P_{4}\right)=4$ and $\operatorname{adrn}\left(P_{5}\right)>r n\left(P_{5}\right)=3$. However, $\operatorname{adrn}\left(K_{1,3}\right)=r n\left(K_{1,3}\right)=3$.

We summarize our results on the adrn and the corresponding results on the $d r n$ in Table 1.

\section{Acknowledgments}

The second author is very grateful to Professor S. Ramachandran for drawing his attention to $\operatorname{adrn}(G)$. The authors are thankful to anonymous referees for their many valuable comments which largely improved the style of the paper and the proof of Theorem 4 . The work reported here is supported by the Project SR/S4/MS:628/09 awarded to the first author by the Department of Science and Technology, Government of India, New Delhi.

\section{References}

[1] F. Harary, Graph Theory, Addison-Wesley, Reading, Mass, USA, 1969.

[2] S. M. Ulam, A Collection of Mathematical Problems, Wiley, New York, NY, USA, 1960.

[3] F. Harary and M. Plantholt, "The graph reconstruction number," Journal of Graph Theory, vol. 9, no. 4, pp. 451-454, 1985.
[4] W. Myrvold, "The ally-reconstruction number of a disconnected graph," Ars Combinatoria, vol. 28, pp. 123-127, 1989.

[5] W. J. Myrvold, The ally and adversary reconstruction problems [Ph.D. thesis], University of Waterloo, 1988.

[6] P. K. Stockmeyer, "The falsity of the reconstruction conjecture for tournaments," Journal of Graph Theory, vol. 1, no. 1, pp. 1925, 1977.

[7] S. Ramachandran, "Degree associated reconstruction number of graphs and digraphs," Mano International Journal on Mathematical Sciences, vol. 1, pp. 41-53, 2000.

[8] M. D. Barrus and D. B. West, "Degree-associated reconstruction number of graphs," Discrete Mathematics, vol. 310, no. 20, pp. 2600-2612, 2010.

[9] S. Sundar Raj and S. Monikandan, "Adversary degree associated reconstruction number of graphs obtained from complete graphs or cycles," Journal of Combinatorics, Information \& System Sciences, vol. 37, no. 1, pp. 75-94, 2012. 


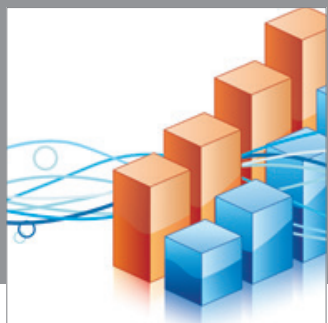

Advances in

Operations Research

mansans

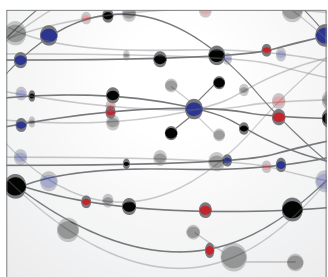

The Scientific World Journal
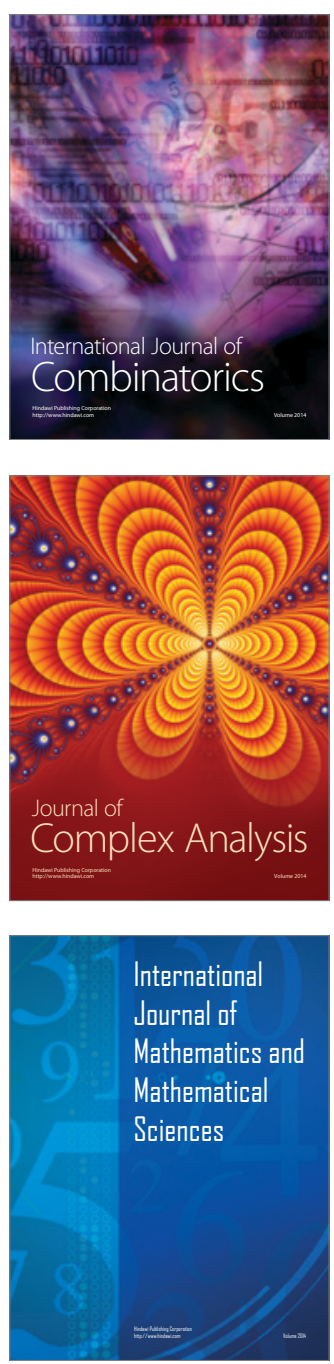
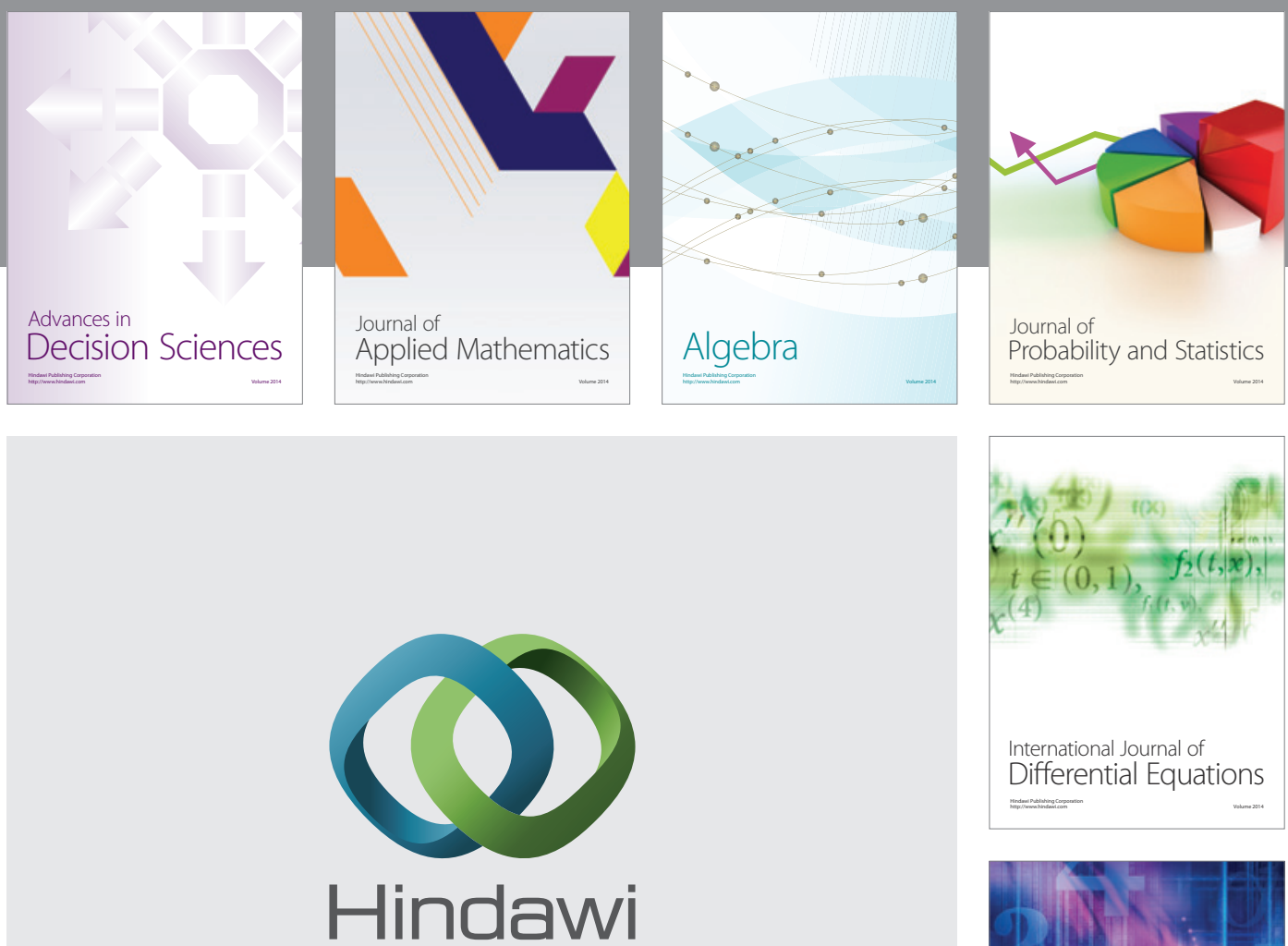

Submit your manuscripts at http://www.hindawi.com
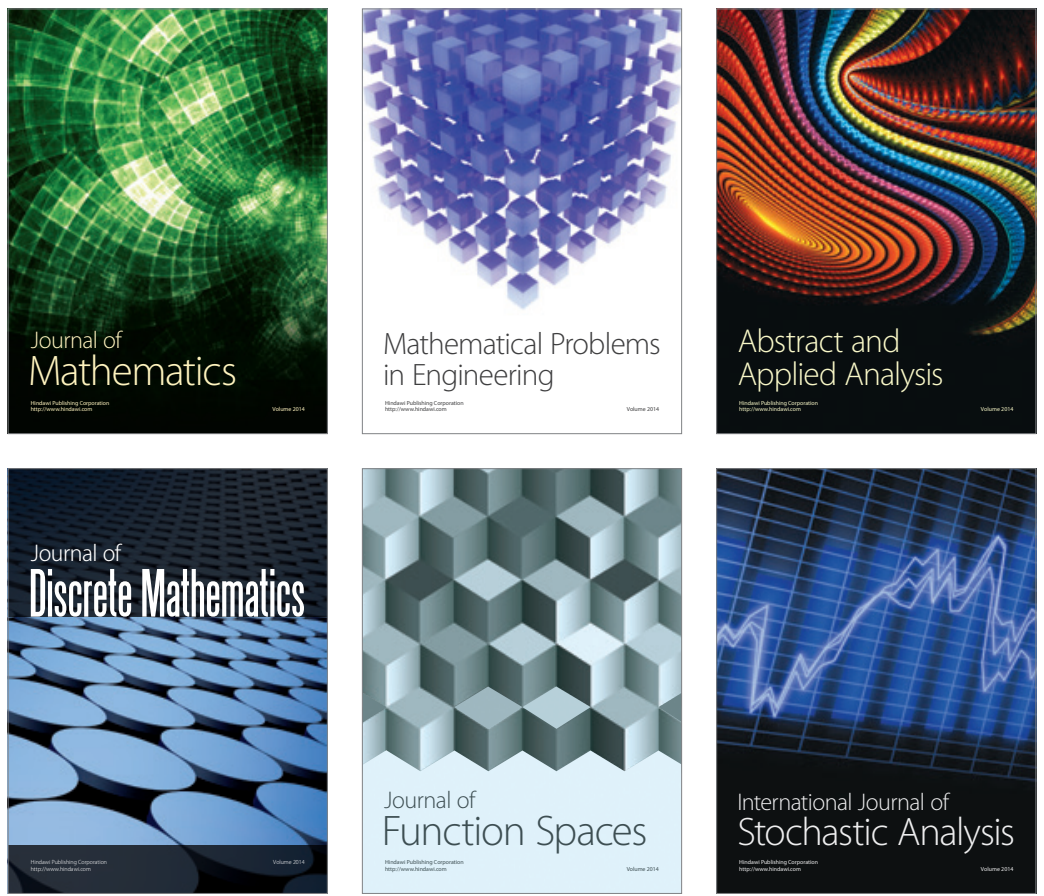

Journal of

Function Spaces

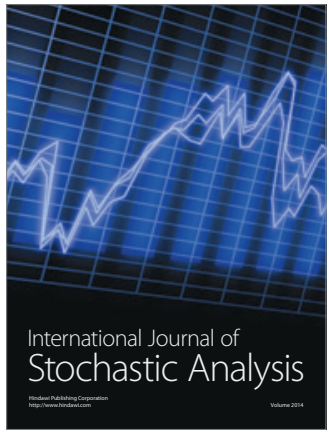

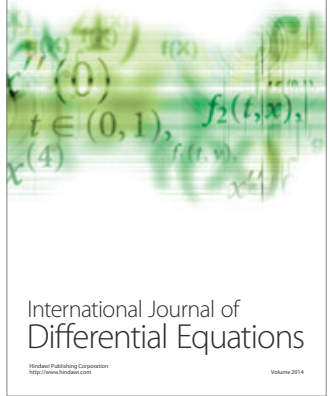
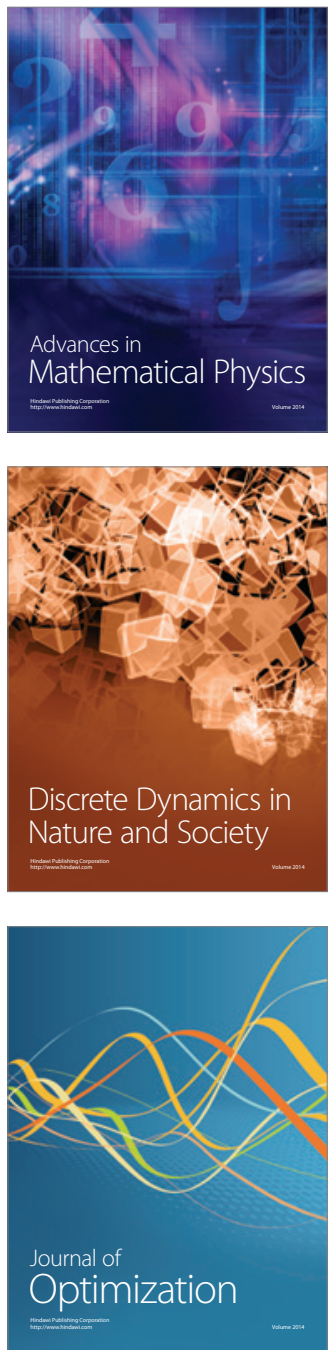